\title{
ASPECTOS DA ALIMENTAÇÃO DE BRYCONAMERICUS MICROCEPHALUS (CHARACIFORMES, TETRAGONOPTERINAE) NO CÓRREGO ANDORINHA, ILHA GRANDE - RJ
}

\author{
Carla Ferreira Rezende ${ }^{1}$ \& Rosana Mazzoni ${ }^{2}$
}

Biota Neotropica v3 (n1) - http://www.biotaneotropica.org.br/v3n1/pt/abstract?short-communication+BN01603012003

\author{
Recebido em 03/02/2003 \\ Revisado em 13/03/2003 \\ Publicado em 08/04/2003 \\ ${ }^{1}$ Laboratório de Ecologia de Peixes - IBRAG/UERJ / Ecologia \\ Av. São Francisco Xavier 524, RJ -20550-013 \\ email: carla.fr@terra.com.br \\ ${ }^{2}$ Laboratório de Ecologia de Peixes - IBRAG/UERJ / Ecologia \\ Av. São Francisco Xavier 524, RJ -20550-013 \\ email: mazzoni@uerj.br
}

\begin{abstract}
Astract
The present study was developed at córrego Andorinha in the oceanic slope of Ilha Grande $\left(23^{\circ} 05^{\prime}, 23^{\circ} 15^{\prime} \mathrm{S}\right.$ e $44^{\circ} 06$ e $44^{\circ} 23^{\prime} \mathrm{W}$ ) and aimed to determine $B$. microcephalus feeding habits as well as their differences between adult and juvenile specimens. Sampling were carried out between Nov/99 and Oct/00, by waiting nets (5-10 mm mesh) and sieves in a stream strech characterized by $100 \%$ of canopy and sand, gravel, rocks and debris substrata. At each sampling ocasion, 20 specimens were collected and their gut content analysed under stereoscopic microscopic following the Numerical Frequency method. Diet was based mainly on aquatic insect larvaes (autochthonous) and terrestrial insects (allochthonous). The participation of allochthonous and autochthonous itens varied seasonally $\left(\mathrm{r}_{\text {spearman }}=0.296 ; \mathrm{p}=0.325\right)$, being allochthonous itens predominant during dry season. Considering the relative participation of allochthonous and autochthonous itens in the diet of juvenile and adult, separatelly, we found predominance of allochthonous itens among juveniles ( $t$ test; $t=2.51$, $\mathrm{df}=30 ; \mathrm{p}=0.01$ ) and absence of differences among adult specimens ( $\mathrm{t}$ test; $\mathrm{t}=0.79, \mathrm{df}=84 ; \mathrm{p}=0.43$ ). Mean value of Intestinal Coefficient (IC) was 0.77, without differences among juveniles and adult specimens ( $\mathrm{t}$ test; $\mathrm{t}=-0.62, \mathrm{df}=85$; $\mathrm{p}=0.45$ ), and suggests an omnivorous diet, that was not corroborated by the registered diet. Such result should be explained by a pontual sample that was proceeded in a low primary production locality (100\% canopy cover); nonetheless, as migrators, this specimens must use other food resources (e.g. filamentous algae and/or aquatic macrophytes) in the localities where primary production is enhanced by sun incidence (open areas), explaning their anatomical pre-adaptation (omnivorous CI). Key Words: Atlantic Forest, Tetragonopterinae, feeding habit, Bryconamericus
\end{abstract}

\section{Resumo}

Esse estudo foi realizado no Córrego Andorinha, localizado na vertente oceânica da Ilha Grande $\left(23^{\circ} 05^{\prime}, 2^{\circ} 15^{\prime} \mathrm{S}\right.$ e $44^{\circ} 06$ e $44^{\circ} 23^{\prime} \mathrm{W}$ ) e teve como objetivo determinar a dieta de B. microcephalus, bem como suas variações entre jovens e adultos. As coletas foram realizadas entre nov/99 e out/00 através de rede de espera e arrastinho (malhas $5 \mathrm{~mm}$ e $10 \mathrm{~mm}$ ) em um trecho do riacho onde a vegetação marginal cobre $100 \%$ da localidade, o substrato é formado por areia, cascalho, rochas e folhiço. A cada ocasião de coleta 20 exemplares eram amostrados e o conteúdo estomacal analisado sob microscópio estereoscópico através do método da Frequiência Numérica. A dieta da espécie é baseada em larvas de insetos aquáticos (autóctones) e insetos terrestres (alóctones). A participação dos itens autóctones e alóctones variam sazonalmente $\left(\mathrm{r}_{\text {Spearman }}=0,296 ; \mathrm{p}=0,325\right)$ sendo que os ítens alóctones predominaram na estação seca. Considerando-se a importância relativa dos itens alóctones e autóctone na dieta de jovens e adultos, separadamente, observamos predomínio de itens alóctones entre os primeiros (teste $\mathrm{t} ; \mathrm{t}=2,51 ; \mathrm{gl}=30 ; \mathrm{p}=0,018$ ) e ausência de diferença entre os adultos (teste $\mathrm{t} ; \mathrm{t}=0,79 ; \mathrm{gl}$ $=84 ; \mathrm{p}=0.429)$. O valor médio do Quociente Intestinal $(\mathrm{QI})$ foi 0,77 , sem diferenças entre jovens e adultos (teste $\mathrm{t} ; \mathrm{t}=-0,62$; $\mathrm{gl}=85 ; \mathrm{p}=0,45)$, e sugere dieta onívora fato não corroborado pela dieta registrada. Isso pode ser explicado pela pontualidade das amostras realizadas em uma localidade de baixa produção primária decorrente da baixa insolação (100\% cobertura); no entanto, como migradores, esses exemplares certamente utilizam outros recursos (e.g. algas filamentosas e/ou vegetais superiores) em outras localidades onde a produção primária é incrementada pela insolação (áreas abertas), justificando a pré-adaptação anatômica da população (QI de onívoro).

Palavras chaves: Mata Atlântica, Tetragonopterinae, hábito alimentar, Bryconamericus 


\section{INTRODUÇÃO}

A fauna de peixes de água doce da América do Sul é a mais rica e diversificada ictiofauna continental do planeta, reunindo aproximadamente 60 famílias, várias centenas de gêneros e, provavelmente, em torno de 5000 espécies (Vari \& Weitzman, 1990). Em uma avaliação mais recente, Vari \& Malabarba (1998) sugerem que a ictiofauna de água doce sul americana é composta por aproximadamente 8000 espécies e que $65 \%$ desse total não está descrito e, portanto, desconhecido da ciência.

Dentre os diferentes sistemas fluviais que comportam a referida diversidade de espécies, destacamos os riachos costeiros da Serra do Mar (Mata Atlântica) que formam um corredor de aproximadamente $3000 \mathrm{~km}$ ao longo da costa leste do Brasil (Bacia do Leste) e, segundo Bizerril (1994), comporta aproximadamente $10 \%$ do total das espécies citadas anteriormente, indicando a sua importância para o patrimônio ictiofaunístico da Mata Atlântica.

Atualmente, apenas 5\% da vegetação original da Mata Atlântica encontra-se intacta, determinando que os sistemas naturais desta região estão em franca modificação (Coltrinari, 1996) com muitas espécies em sério risco de desaparecimento (Menezes et al., 1990; Mazzoni et al., 2000). Esse tipo de informação, associado à elevada taxa de perda de ambientes naturais (desflorestamento) aponta para a importância de estudos que viabilizem o entendimento da dinâmica das populações e comunidades.

Neste contexto, estudos sobre alimentação de peixes são fundamentais por fornecerem dados para a formulação de modelos sobre a estrutura trófica dos ecossistemas e conhecimentos quantitativos dos mecanismos biológicos de interação entre as espécies, tais como predação e competição (Zavala-Camin, 1996). A necessidade de estudos sobre a biologia das espécies de peixes da Ilha Grande tem como fator principal a ausência de informações, desse tipo, para a região. Em recente levantamento da ictiofauna de alguns sistemas fluviais das vertentes continental e oceânica, foram registradas nove espécies de peixes de água doce, sendo que Bryconamericus microcephalus se destaca como a espécie de maior distribuição e abundância (Mazzoni et al., 2001). As demais populações de peixes, presentes nos cursos d'água da ilha, são extremamente rarefeitas, ressaltando a importância de estudos sobre sua história de vida.

\section{ÁREA DE ESTUDO}

O presente estudo foi realizado em uma localidade

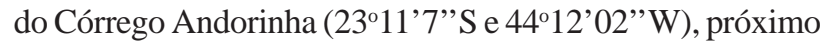
ao Centro de Estudos Ambientais e Desenvolvimento Sustentável (CEADS/UERJ) na vertente oceânica da Ilha Grande ( $23^{\circ} 05^{\prime} \mathrm{e} 23^{\circ} 15^{\prime} \mathrm{S}, 44^{\circ} 06$ e $\left.44^{\circ} 23^{\prime} \mathrm{W}\right)$, localizada na Baía da Ilha Grande, município de Angra dos Reis, Rio de Janeiro. A área de estudo situa-se em uma região de Mata
Atlântica primária, de clima quente e úmido, com precipitação média de aproximadamente $1500 \mathrm{~mm}$ e temperatura de $22,5^{\circ} \mathrm{C}$, oscilando entre $25,7^{\circ} \mathrm{C}$ (fevereiro) e $19,6^{\circ} \mathrm{C}$ (julho) (Oliveira \& Neto, 1996).

O sistema fluvial da Ilha Grande é composto por uma série de riachos de pequeno e médio porte, que vertem tanto para a região oceânica (Fig. 1) como para a região continental (Fig. 2) e possuem inúmeras áreas onde a drenagem é subterrânea. O local de coleta compreende um trecho de rio, de aproximadamente $150 \mathrm{~m}$ de extensão, no interior de mata de galeria formando um dossel fechado com $100 \%$ de cobertura vegetal (Fig. 3). Caracteriza-se por uma região de rápidos intercalados por remansos laterais e substrato formado por areia $(80 \%)$, cascalho $(10 \%)$, rochas $(5 \%)$ e folhiço.

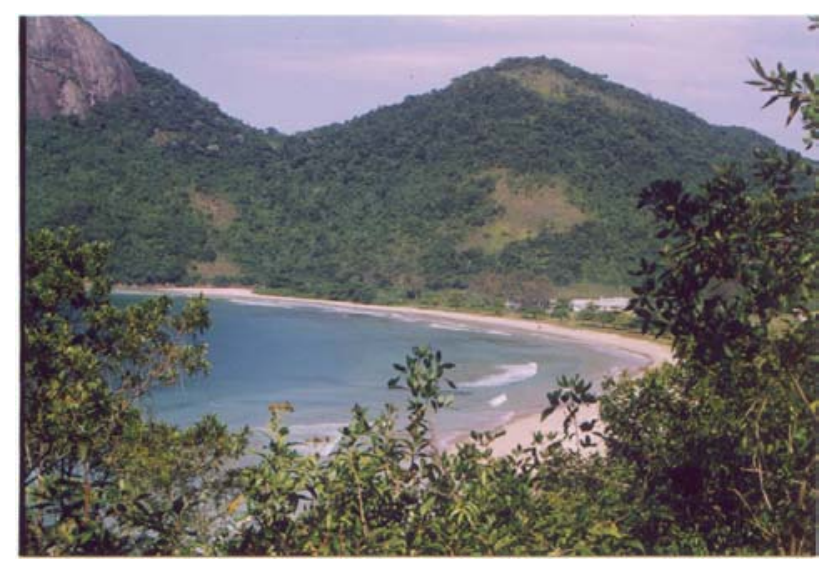

Fig. 1. Vista panorâmica da vertente oceânica da Ilha Grande.

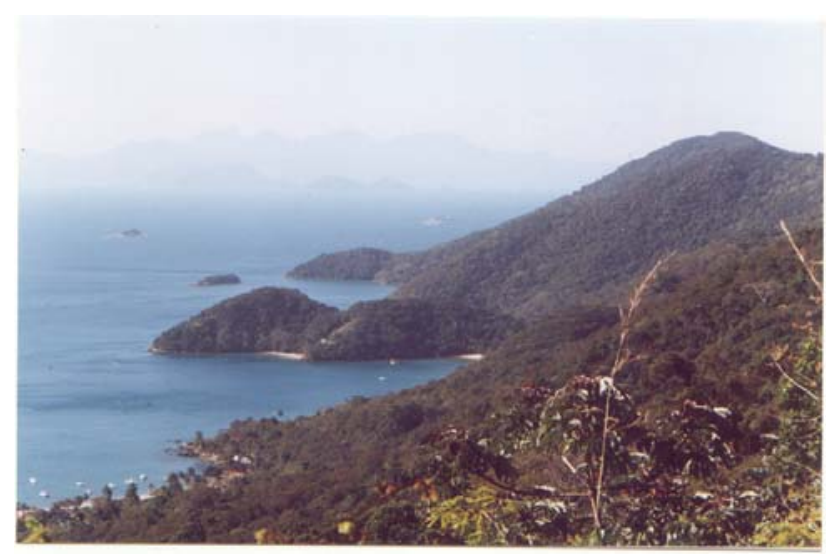

Fig. 2. Vista panorâmica da vertente continental da Ilha Grande. 


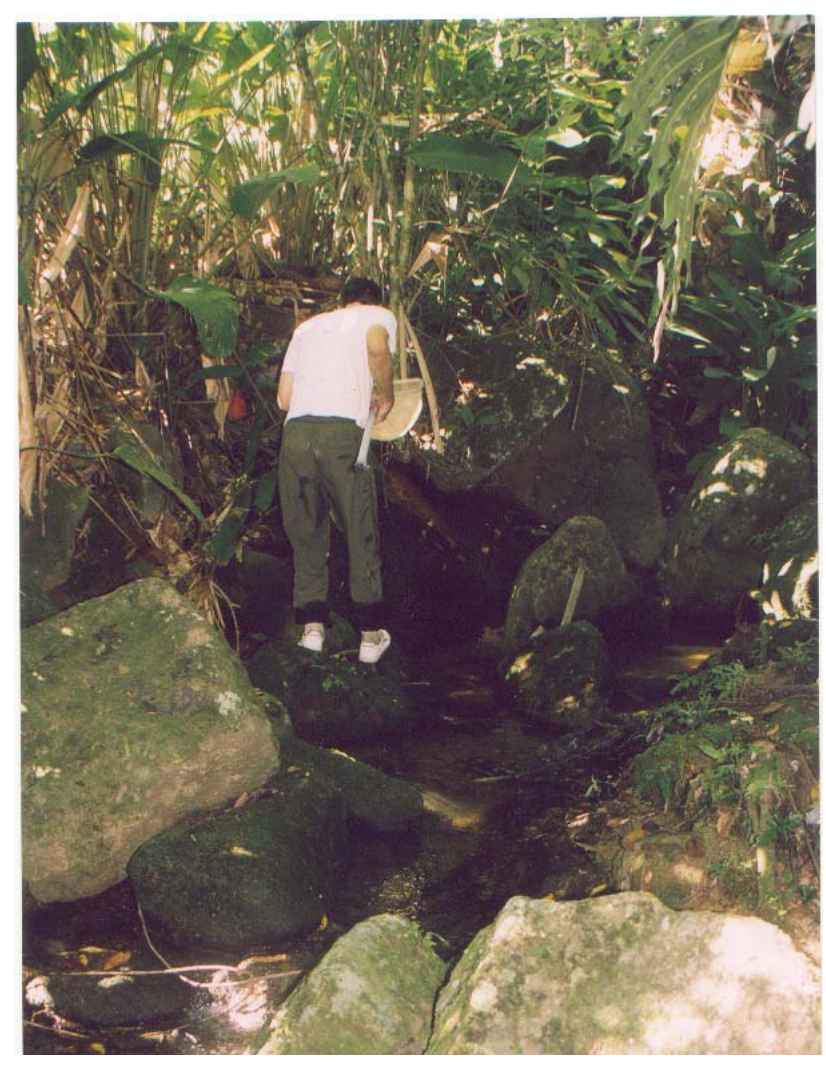

Fig. 3. Detalhe do trecho amostrado no córrego Andorinha, Ilha Grande.

\section{MATERIAIS E MÉTODOS}

Coletas mensais foram realizadas no Córrego Andorinha no período de novembro de 1999 a outubro de 2000, através de redes de espera (malha $10 \mathrm{~mm}$ ), arrastinho (malha $5 \mathrm{~mm}$ ) e peneiras. Os exemplares amostrados foram mantidos em gelo e transportados ao laboratório para registro dos dados de comprimento padrão $(\mathrm{CP}, \mathrm{cm})$, peso total (PT, g) e comprimento do intestino (CI, cm). O estômago de cada exemplar coletado foi retirado e fixado em formol $5 \%$ para posterior análise. Exemplares-testemunho de B. microcephalus (Fig. 4), encontram-se disponíveis na coleção de referência do Laboratório de Ecologia de Peixes da Universidade do Estado do Rio de Janeiro.

A análise do conteúdo estomacal foi realizada através

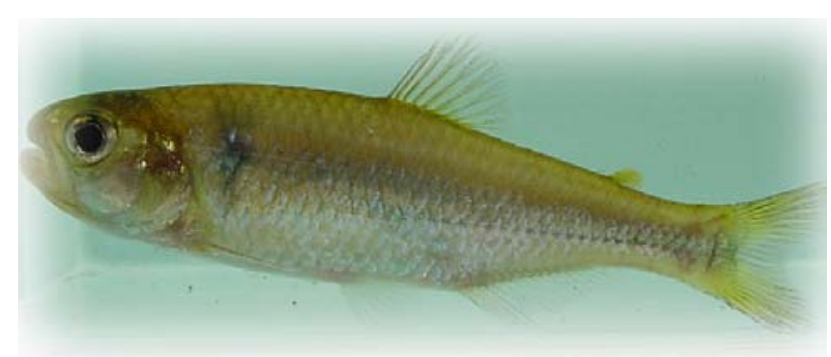

Fig. 4. Exemplar de Bryconamericus microcephalus $(6,2 \mathrm{CP} ; \mathrm{cm})$ coletado do córrego Andorinha. do método da Freqüência Numérica (Hyslop, 1980) mediante estereomicroscópio; análises qualitativas complementares foram realizadas através de microscopia ótica. A identificação dos itens alimentares seguiu a bibliografia especializada (Buzzi \& Miyazaki, 1999; Merritt \& Cummins, 1984; Trivinho-Strixino, 1995). O quociente intestinal (QI), utilizado como acessório para a caracterização do hábito alimentar da espécie (Barbieri, 1994), foi calculado de acordo com o modelo a seguir: $\mathrm{QI}=\mathrm{CI} / \mathrm{CP}$, onde $\mathrm{CI}=$ comprimento do intestino e $\mathrm{CP}=$ comprimento padrão e a significância das diferenças de seus valores médios entre jovens e adultos foi determinada pelo teste t. O estabelecimento das classes de tamanho que reúnem os exemplares jovens e adultos, foi realizada com base em Fagundes \& Mazzoni (2003).

A participação dos itens alimentares de origem autóctone e alóctone foi avaliada para jovens e adultos e as diferenças testadas pelo teste t. Considerando que os principais eventos sazonais da região se caracterizam pelos períodos secos e chuvosos, e a fim de investigar a influência de tal sazonalidade na dieta da espécie, agrupamos os dados relativos aos 12 meses de coleta em duas estações: seca (abril a setembro) e chuvosa (outubro a março). Para tal foi estimada a média sazonal das frequiências numéricas dos itens quantificáveis; análises de correlações nãoparamétricas (Spearman) foram utilizadas para detectar possíveis variações sazonais das freqüências numéricas médias dos diferentes itens alimentares.

\section{RESULTADOS}

Durante o período de estudo foram coletados 105 exemplares de $B$. microcephalus, sendo que durante os meses de julho e agosto de 2000 nenhum exemplar foi registrado na área de estudo. As análises referentes ao conteúdo estomacal de B. microcephalus foram baseadas em 89 estômagos e indicam predomínio de larvas aquáticas de insetos (insetos autóctones) e formas adultas de insetos terrestres (insetos alóctones). Na Tab. I são apresentadas as freqüências numéricas médias dos diferentes itens que compõem a dieta da espécie.

A participação percentual da matéria de origem autóctone e alóctone na dieta de $B$. microcephalus variou segundo a sazonalidade $\left(\mathrm{r}_{\text {Spearman }}=0,296, \mathrm{p}=0,325\right)$. Na estação seca observou-se predomínio de itens alóctones em relação aos itens autóctones; na estação chuvosa não houve diferença na importância desses dois tipos de alimento, tendo sido registradas freqüências semelhantes de artrópodes de origem autóctone e alóctone (Tab. I).

Não foram registradas diferenças significativas no QI médio de jovens e adultos de $B$. microcephalus (teste t; $\mathrm{t}=-0,62 ; \mathrm{gl}=85 ; \mathrm{p}=0,45-$ Fig. 5), sendo registrado um QI de 0,77 . No que se refere ao consumo de itens de origem alóctone e autóctone entre exemplares jovens e adultos, observamos diferenças significativas com predomínio de

http://www.biotaneotropica.org.br 
itens alóctones entre os jovens (teste $\mathrm{t} ; \mathrm{t}=2,51 ; \mathrm{gl}=30 ; \mathrm{p}=$

0,018 ) e ausência de diferenças (teste $\mathrm{t} ; \mathrm{t}=0,79 ; \mathrm{gl}=84 ; \mathrm{p}=$

0.429) entre os adultos (Fig. 6).

Tabela I. Freqüência numérica média dos itens alimentares registrados nos estômagos de Bryconamericus microcephalus, de acordo com as estações de seca e chuva.

\begin{tabular}{|c|c|c|c|c|c|c|}
\hline \multirow[t]{2}{*}{ Matéria Autóctone } & & \multicolumn{4}{|c|}{ Matéria Alóctone } & \multirow[b]{2}{*}{ Seca } \\
\hline & & Chuva & Seca & & Chuva & \\
\hline Artrópodos Imaturos & & & & Artrópodos Adultos & & \\
\hline Larva de Coleoptero & & 0,04 & 0,05 & Aracnídeo & 0,15 & 0,00 \\
\hline Larva de Díptero & & 1,36 & 0,71 & Colembola & 0,14 & 0,00 \\
\hline Larva de Odonato & & 0,16 & 0,00 & Coleóptero & 0,18 & 0,31 \\
\hline \multirow[t]{2}{*}{ Larva de Efemeróptero } & & 0,20 & 0,00 & Díptero & 0,41 & 0,53 \\
\hline & total & 1,76 & $\mathbf{0 , 7 6}$ & Hemíptero & 0,10 & 0,10 \\
\hline Artrópodos Adultos & & & & Himenóptero & 0,70 & 0,31 \\
\hline \multirow[t]{3}{*}{ Copépode } & & $\mathbf{0 , 0 8}$ & $\mathbf{0 , 0 0}$ & Homóptero & 0,22 & 0,40 \\
\hline & & & & Tricóptero & 0,32 & 0,16 \\
\hline & & & & & Total 2,22 & 1,81 \\
\hline
\end{tabular}

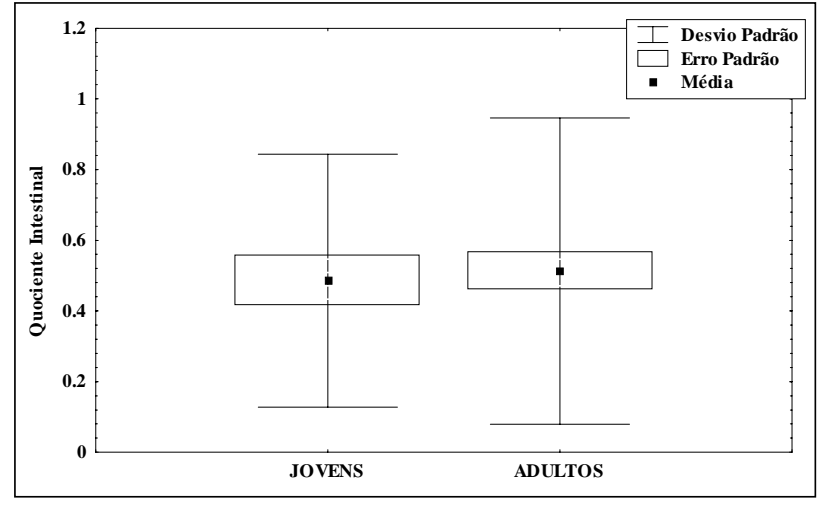

Fig. 5. Valores médios do quociente intestinal (QI) de exemplares jovens e adultos de Bryconamericus microcephalus.

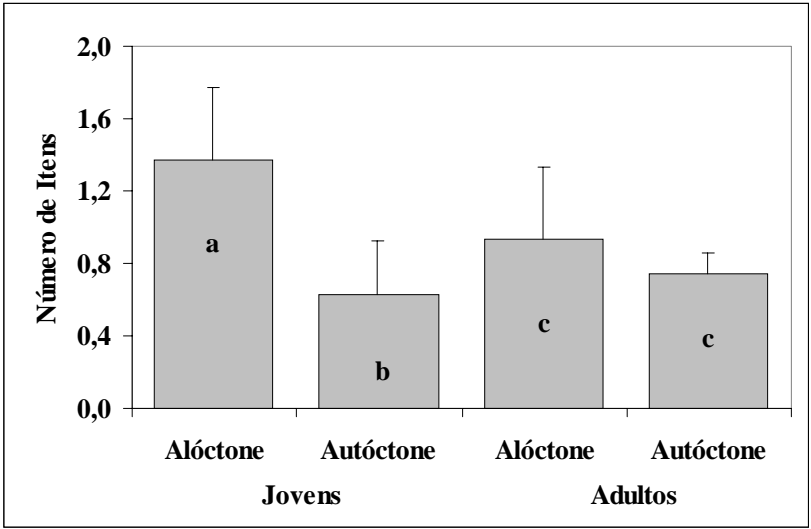

Fig. 6. Valores médios da freqüência numérica dos itens alimentares autóctones e alóctones ingeridos por Bryconamericus microcephalus. As barras indicadas com a mesma letra representam ausência de diferenças significativas. 


\section{DISCUSSÃO}

Observamos que durante o período de estudo não foram coletados exemplares nos meses de julho e agosto (inverno). A principal causa desse fato pode ser atribuída ao hábito migrador da espécie que, possivelmente, durante o período de inverno (seca) empreende movimentos mais restritos, não estando disponível na localidade de estudo.

O quociente intestinal de B. microcephalus indica dieta onívora, corroborando trabalhos anteriores relativos a esse gênero (Escalante, 1987; Uieda et al., 1997; Casatti \& Castro, 1998). No entanto, as análises do conteúdo estomacal não indicam presença de material de origem vegetal como componente da dieta. Estudos paralelos sobre o aporte de matéria alóctone vêm sendo realizados na mesma localidade do presente estudo, e indicam ausência de aporte de matéria vegetal, tal como frutos e sementes, que poderia ser um recurso utilizado pela espécie (dados não publicados). Considerando que a localidade de estudo é $100 \%$ sombreada por vegetação de cobertura, sugere-se que a produção primária local é limitada em função da baixa incidência luminosa. Essa circunstância, associada à não disponibilidade de frutos e sementes limita localmente a disponibilidade de recursos de origem vegetal e pode ser uma explicação para a dieta essencialmente carnívora dos exemplares estudados.

Nesse contexto, ressaltamos que nossos resultados são limitados a uma localidade com características peculiares. Por ser um ambiente densamente coberto por vegetação de grande porte, tem-se uma área sombreada com reduzida produção primária. Com isso, os exemplares coletados possuem dieta predominantemente composta por insetos de origem autóctone e alóctone fato que, no entanto, não descarta a possibilidade de se alimentarem de matéria vegetal em outro trecho do rio, visto tratar-se de uma espécie migradora e oportunista (Mazzoni \& Iglesias, 2002); isso explicaria o QI típico de espécie onívora.

Russo \& Hahn (2001) verificaram que em duas espécies do gênero de Bryconamericus havia importante segregação na utilização dos recursos alimentares, sendo que uma espécie consumia basicamente formas larvais de insetos enquanto que a outra consumia formas adultas de insetos. Esse tipo de resultado sugere plasticidade da espécie, no que se refere ao uso dos recursos alimentares. Hábitos alimentares oportunistas em representantes da subfamília Tetragonopterinae são amplamente citados na literatura. Delariva et al., (2001) observaram que diferentes espécies de Astyanax podem mudar seus hábitos insetívoros para herbívoros, após determinadas modificações no ambiente. No caso de Astyanax sp., no reservatório de Segredo, houve alternância da dieta exclusivamente herbívora para um consumo de insetos relativo à mais de $70 \%$ da dieta (Vargas et al., 2001). Comparando alimentação da espécie Astyanax scabripinnis em dois riachos da Bacia do Rio Tibagi, Oricalli et al. (2001) encontraram resultados que indicam oportunismo na exploração do material autóctone e alóctone, dependendo da característica de cada riacho analisado.

As diferenças qualitativas sazonais na composição da dieta de $B$. microcephalus podem estar relacionadas ao ciclo de vida dos artrópodes consumidos por esta espécie. Muitos peixes apresentam plasticidade em suas dietas. Predadores mudam de presas de acordo com o seu crescimento, mudança de biótipo ou com a disponibilidade de alimento de acordo com a sazonalidade (LoweMcConnell, 1987).

Dessa forma, sugerimos que a dieta de B. microcephalus do córrego Andorinha está fortemente determinada pelas características locais e, conseqüentemente, pelo tipo de recursos disponíveis. No entanto, por ser uma espécie que empreende movimentos migratórios de subida e descida, é possível que exemplares obtidos em outras localidades com características fisionômicas distintas apresentem dieta diferente da registrada nesse trabalho. Ressaltamos, portanto, a importância do prosseguimento desse estudo, a fim de reunirmos resultados mais consistentes sobre o espectro alimentar da espécie.

\section{REFERÊNCIAS BIBLIOGRÁFICAS}

BARBIERI, G., PERET, A.C. \& VERANI, J.R. 1994. Notas sobre a alimentação do trato digestivo ao regime alimentar em espécies de peixes da região de São Carlos (SP) I. Quociente Intestinal. Rev. Brasil. Biol. 54:63-69.

BIZERRIL, C.R.S.F. 1994. Análise taxonômica e biogeográfica da ictiofauna de água doce do leste brasileiro. Acta Leopoldensia 16:51-80.

BUZZI, Z. J. \& MIYAZAKI, D.R. 1999. Entomologia didática. 3 ed. Editora da UFPR, Curitiba.

CASATI, L. \& CASTRO, R.M.C. 1998. A fish community of the São Francisco River headwater riffles, southeastern Brazil. Ichthyol. Explor. Freshwaters 9:229-242.

COLTRINARI, L. 1996. Natural and anthropogenic interactions in the Brazilian tropics. In: Geoindicators. Assessing rapid environmental changes in earth systems. (eds. Berger, A.R. \& Aims, W.J.). A.A. Balkema Publ. Rotterdan, The Netherlands.

DELARIVA, R.L.; LOUREIRO, V.E.; CASSEMIRO, F.A.S.; HAHN, N.S. \& CARNEIRO, G. 2001. Partição temporal de recursos alimentares entre espécies de Astyanax (Characidae, Tetragonopterinae) no Rio Iguaçu-PR, Brasil. Resumos do XIV Encontro Brasileiro de Ictiologia.

ESCALANTE, H. A. 1987. Alimentacion de Bryconamericus ihering y Jenynsia lineata_lineata (Osteichtyes) en Sierra de La Ventana (Argentina). An. Mus. Hist. Nat. Valparaíso, 18:101-108. 
FAGUNDES, A.P. \& MAZZONI. R. 2003. Aspectos da história de vida e reprodução de Bryconamericus microcephalus, no Córrego Andorinha, Ilha Grande, RJ. Resumos XV Encontro Brasileiro de Ictiologia. Universidade Presbiteriana Mackenzie/SBI, São Paulo.

HYSLOP, E.J. 1980. Stomach contents analysis - a review of methods and their application. J. Fish Biol. 17:411-429.

LOWE-McConnell, R.H. 1987. Ecological studies in tropical fish communities. Cambrige University Press, New York, N.Y, p. 270-280.

MAZZONI, R.; FENERICH-VERANI, N. \& CARAMASHI, E.P. 2000. Electrofishing as a sampling technique for coastal stream fish populations in the Southeast of Brazil. Rev. Brasil. Biol. 60:205-216.

MAZZONI, R.; TOSTA, G.C.; PETITO, J.; MIRANDA, J.C. \& SCHUBART, S.A. 2001. A ictiofauna de água doce da Ilha Grande - RJ. Resumos XIV Encontro Brasileiro de Ictiologia. Unisinos/SBI, São Leopoldo.

MAZZONI, R. \& IGLESIAS, R.R. 2002. Distribution pattern of two fish species in a coastal stream in the southeast of Brazil. Braz. J. Biol. 62:171-178.

MENEZES, N.A.; CASTRO, R.M.C.; WEITZMAN, S.H. \& WEITZMAN, M.J. 1990. Peixes de riacho da floresta costeira Atlântica brasileira: um conjunto pouco conhecido e ameaçado de vertebrados. In II Simpósio de ecossistemas da Costa Sul e Sudeste Brasileira: estrutura, função e manejo. Academia de Ciências do Estado de São Paulo, v. I, p. 290-295.

MERRITT, W.R. \& CUMMINS, K.W. 1984. An introduction to the aquatic insects of North America, Kendall/Hunt Publishing Company, Dubukue, Iowa.

OLIVEIRA, R.R. \& NETO, A.L.C. 1996. Os rastros do homem na floresta. Albertoa 4:109-116.

ORICALLI, M.C.G.; TEIXEIRA, I. \& BENNEMANN, S.T. 2001. Dieta de Astyanax scabripinnis coletados em dois riachos da Bacia do Rio Tibagi, PR. Resumos do XIV Encontro Brasileiro de Ictiologia. Unisinos/SBI, São Leopoldo.

RUSSO, M.R. \& HAHN, N.S. 2001. Ecologia trófica de duas espécies de Bryconamericus (Characidae, Tetragonopterinae) do Rio Iguaçu-PR, Brasil. Resumos do XIV Encontro Brasileiro de Ictiologia. Unisinos/SBI, São Leopoldo.

TRIVINHO-STRIXINO, S. \& STRIXINO, G. 1995. Larvas de Chironomidae (Diptera) do Estado de São Paulo. Guia de identificação e diagnose dos gêneros. PPG-ERN/ UFSCAR, São Carlos.

VARGAS, R.D.; ABUJANRA, F.; FUGI, R. \& AGOSTINHO, A. 2001. Ecologia alimentar de duas espécies de lambari do Reservatório de Segredo após o terceiro ano de formação. Resumos do XIV Encontro Brasileiro de Ictiologia. Unisinos/SBI, São Leopoldo.
VARI, R.P. \& WEITZMAN, S.H. 1990. A review of the phylogenetic biogeography of the freshwater fishes of the South America. In Vertebrates in the Tropics. (G. Peters, R. Hutterer, eds.) In: Proceedings of the International Symposium on Vertebrate Biogeography and Systematics in the Tropics, Bonn, june 1989. Alexander Koenig Zoological Research Institute and Zoological Museum, p. 381-393.

VARI, R. \& MALABARBA, L.R. 1998. Neotropical ichthyology: an overview. In Phylogeny and Classification of Neotropical Fishes. (L.R. Malabarba; R.E. Reis; R.P. Vari; Z.M.S. Lucena; C.A.S. Lucena eds.). Porto Alegre: EDIPUCRS, p. 1-11.

UIEDA, V.S; BUZZATO, P. \& KIKUCHI, R.M. 1997. Partilha de recursos alimentares em peixes em um riacho de serra do Sudeste do Brasil. An. Acad. Bras. Ci. 69:243-252.

ZAVALA-CAMIN, L.A. 1996. Introdução ao estudo sobre alimentação natural em peixes. EDUEM, Maringá.

Título: Aspectos da alimentação de Bryconamericus microcephalus (Characiformes, Tetragonopterinae) no Córrego Andorinha, Ilha Grande - RJ

Autores:Carla Ferreira Rezende \& Rosana Mazzoni

Biota Neotropica, Vol. 3 ( number 1): 2003

http://www.biotaneotropica.org.br/v3n 1/pt/ abstract?short-communication+BN01603012003

Recebido em 03/02/2003 Revisado em 13/03/2003 Publicado em 08/04/2003

ISSN 1676-0603

http://www.biotaneotropica.org.br 